\title{
What is the impact of physician communication and patient understanding in the management of headache?
}

\author{
Meenal Patwardhan' \\ Remy R Coeytaux ${ }^{2}$ \\ Rajeshwari Deshmukh ${ }^{3}$ \\ Gregory Samsa ${ }^{4}$ \\ 'Department of Medicine, Duke \\ University Medical Center, Durham, \\ NC, USA; ${ }^{2}$ Department of Family \\ Medicine, University of North \\ Carolina, Chapel Hill, NC, USA; \\ ${ }^{3}$ Department of Rheumatology, \\ Oregon Health Sciences University, \\ Portland, OR, USA; ${ }^{4}$ Department of \\ Biostatistics and Bioinformatics, Duke \\ University, Durham NC, USA
}

Correspondence: Meenal B Patwardhan Duke Center for Clinical Health Policy Research, 2200 W. Main St., Suite 220, Durham NC 27705, USA

$\mathrm{Tel}+\mid 9192863399$

$\mathrm{Fax}+19192865601$

Email meenal.p@duke.edu

\begin{abstract}
Migraine is a common and debilitating condition. Despite the burden of disease and increasing availability of effective treatment, migraine management is unsatisfactory. Evidence in other chronic conditions indicates that effective physician communication results in better patient understanding and health outcomes.

The current literature review was intended to evaluate evidence regarding the relationship of effective physician-provider communication to health outcomes and patient satisfaction among patients with migraine. The authors searched MEDLINE ${ }^{\circledR}$ (1966-June 2007) and the Cochrane Database of Systematic Reviews for relevant publications. The search strategy combined the concepts of "headache disorders" and "physician-patient relations". 912 abstracts were identified, and $80(9 \%)$ of them were included for data abstraction.

There were no studies that met our eligibility criteria. Therefore we revised the eligibility criteria to allow for the inclusion of non-migraine primary headache disorders or the role of non-physician health care providers. Twelve published papers met the revised criteria. The findings from the limited evidence available suggests, but does not prove, that improvements in physician-patient communication could result in a significant decrease in the burden of suffering and health care resource utilization associated with migraine. More research is needed to assess the explicit role of physician-patient communication in the management of migraine.

Keywords: communication, migraine, headache, outcomes, physician communication, patient understanding
\end{abstract}

\section{Background}

Migraine is a common and debilitating condition which affects approximately $12 \%$ of the US population. Half of the individuals with migraine suffer significant impairment in their daily activities and limitation in their productivity at work, school and home (Stewart et al 1992; Lipton, Diamond et al 2001; Lipton, Stewart et al 2001). Despite the evident burden of disease and increasing availability of effective treatment, the management of migraine remains less than satisfactory. (Silberstein and Rosenberg 2000; Edmeads et al 2001; Lipton, Diamond et al 2001; Lipton, Stewart et al 2001). While physicians who manage migraine are motivated to provide optimal headache management, they are constrained by the limited time available for consultation and multiple other medical conditions that compete for physician attention (Lipton et al 1998; South and Sheftell 2001). Ineffective communication (as perceived and reported by patients) has been shown to be associated with dissatisfaction with care and poor adherence to prescribed treatments among headache patients (Cottrell et al 2002). There is also evidence that physician communication regarding a treatment plan, along with distribution of educational materials, results in better patient understanding and better health outcomes (Cottrell et al 2002; Foley et al 2005). 
We sought to evaluate the state of the research that pertains to the relationship of effective physician-provider communication to health outcomes and patient satisfaction among patients with migraine. We conducted a systematic review of the published literature with the objective of identifying research studies that assessed the impact of patient understanding and physician communication on migraine management and patient wellbeing.

\section{Methods}

We searched MEDLINE ${ }^{\circledR}$ (1966-June 2007) and the Cochrane Database of Systematic Reviews for relevant publications. The search strategy combined the concepts of "headache disorders", "physician-patient relations", "communication", "patient education", "health knowledge, attitudes, practice", and "patient understanding". This search was supplemented by a review of the reference lists of included articles.

We did not expect to retrieve a large number of studies that assessed the impact of physician communication and patient understanding in the area of migraine. Therefore, during the process of retrieving literature we also included studies that evaluated (a) the impact of communication (imparted by any individual) and patient understanding in migraine; and (b) the impact of communication and patient understanding in other non-migraine headaches, where no secondary cause was identified. (Note that although we included these studies, our literature search was intended to be comprehensive only for our research question.)

Abstracts of all articles included in the search were independently screened by two reviewers, and a full-text article was obtained if either reviewer selected it for inclusion. Explicit criteria were developed to select full-text articles for data abstraction. We included studies if they were relevant to patient understanding as a result of physician communication, whether they were randomized control trials or observational studies. We excluded articles if the study includes patients with several other diagnoses, and fewer than half of them have a diagnosis of migraine, and if it was a review article.

The two reviewers independently evaluated studies for inclusion using these inclusion criteria. Disagreements were resolved through discussion. Electronic data abstraction forms were developed and piloted for the research question. For each article selected for inclusion, one investigator abstracted relevant data onto the electronic data abstraction form, and the other investigator ascertained the accuracy of the abstracted data.

\section{Results}

We identified 912 abstracts, 80 (9\%) of which were included for data abstraction. The inter-rater agreement for full-text selection of articles was good (kappa of 0.75). When we reviewed these full-text articles we could not retrieve any article that explicitly assessed the impact of physician communication and patient understanding on patient outcomes in migraine. However we identified twelve articles that provided some studies whose results could be extrapolated to our research question.

Eight published articles examined the impact of communication imparted either by a physician in conjunction with other individuals or by a non-physician, on migraine outcomes. A brief description of these studies follows.

Lemstra conducted a randomized control trial, in Canada, that involved 76 patients and compared migraine-related outcomes associated with a multidisciplinary intervention in a non-clinical setting to a control group (Lemstra et al 2002). The intervention included a management approach consisting of exercise, education, lifestyle change and self-management. The multidisciplinary intervention was associated with significant improvement in pain frequency, intensity and duration, as well as functional status, health status, quality of life, health-related disability, and depression. The impact of the individual components of the intervention was not formally assessed. However, patients reported that supervised group exercise sessions, physical therapist's advice, and neurologist's advice and education to be the most effective treatment parameters included in the intervention.

McGrath randomly assigned 73 adolescent migraine patients in Canada to one of three study arms: (a) selfadministered management consisting of an 8-chapter treatment manual and cassette tapes; (b) the same education delivered by a therapist; or (c) a control arm that consisted of education about migraine triggers and instruction on how to use a brainstorming technique to deal with stressful situations (McGrath et al 1992). Both interventions were effective in reducing headaches compared to the control intervention. The clinical gains were maintained after one year.

Holroyd et al (1989) conducted a randomized controlled trial in the USA that compared standard abortive medication therapy administered to 34 recurrent migraine sufferers with and without an educational intervention that promoted selfmanagement. This study demonstrated that self-managed patients had fewer migraine attacks and showed larger reductions in headache activity; both improvements were statistically significant.

Centonze et al (1998) incorporated an intervention, the Cognitive Educational Model, in the medical management of 30 patients with migraine in Italy. The intervention consisted of an educational session delivered by a physician, 
followed by a session in which the patient took notes from the educational session, followed by an explanatory session conducted by an ancillary health care provider. This approach was associated with significant improvements in patients' understanding of migraine as well as a decrease in the number of headache attacks per month and medication use. Outcomes of physician-imparted education were not separately assessed.

Nicholson et al (2005) from USA studied the effectiveness of a self-administered patient-directed intervention that included tailored messages for patients. In a before-after study, the investigators enrolled 33 patients with migraine, and studied the impact of an intervention that included an educational component, skills training component, and weekly tailored messages. Outcomes of the intervention were assessed through an analysis of a daily diaries and self-report questionnaires. Overall $62 \%$ patients reported at least $50 \%$ reduction in headache frequency. Headacherelated disability, behavioral/emotional factors, and headache management self-efficacy also showed significant improvement as a result of the intervention.

Baos et al (2005), in prospective study, conducted in Spain, evaluated the benefits of a structured migraine diary for recording information on response to therapy for a pre-study migraine attack and three consecutive migraine attacks in 97 patients. The investigators claim that patients who used a structured migraine diary reported both improved communication with their doctor about migraine as well as improved satisfaction with their overall medical care provided by their doctor. In addition, most physicians reported that the diary enabled them to better communicate with their patients about migraine, and all reported that it enabled them to assess differences in pain intensity and disability across patients.

Another prospective observational study of 789 patients in USA demonstrated that that the use of appropriate patient and physician educational interventions (that were part of a migraine-management program) improved patient-oriented outcomes and satisfaction (Campinha-Bacote et al 2005).

Rothrock et al (2006) studied the impact of addition of patient education to routine medical management on the clinical status of migraine patients. In a randomized clinical trial setting conducted in USA, 100 patients were randomized to receive or not receive a standardized course of didactic instruction, imparted by trained lay migraineurs) regarding migraine biogenesis and management. The investigators concluded that intensive education of migraine patients by trained lay instructors reduced their mean Migraine Disability
Assessment Scores and may convey significant benefit to migraine patients in terms of reduction in mean headache days per month, functionally incapacitating headache days per month, analgesic overuse and need for abortive therapy (Rothrock et al 2006).

During our search we identified four articles describing results of studies that examined the impact of communication imparted by any individual (irrespective of whether he/she was a physician) in non-migraine headaches disorders. We describe these studies briefly below.

A randomized controlled trial conducted in USA assessed the impact of physician communication on health outcomes in the treatment of chronic headache (Foster et al 2004). The study was designed to evaluate the effectiveness of the Trager approach, which combines manipulative therapy and relaxation training. Thirty-three patients with migraine, tension-type headache, or cluster headache were randomized to one of three arms: (a) medical management of headache without discussion about medication usage (control); (b) medical management in combination with the Trager approach without discussion about medication usage; and (c) medical management plus weekly physician visits for 6-weeks during which medication usage was discussed, patients' headaches over the past week were reviewed, and patients' questions answered. There were no statistically significant differences between the three study groups in terms of the primary headache diary endpoints, but patients randomized to the group that included weekly physician visits demonstrated a decrease in mean headache duration and improved scores on self-reported health-related quality of life as compared to the control group.

Fitzpatrick, from the United Kingdom, interviewed 95 patients ( $53 \%$ had migraine) before and after a new-patient consultation with a neurologist for headache (Fitzpatrick and Hopkins 1981). Thirty-four patients (36\%) reported dissatisfaction with at least one aspect of their doctor's actions; of these, 26 (76\%) reported being dissatisfied with communication from the doctor. Most of these patients cited lack of expansion upon the doctor's diagnosis or "expressed criticism of more specific aspects of the diagnosis that were not explained such as the causes of the diagnosed illness or the implications of the diagnosis for the patient's future life." Patients who reported dissatisfaction with their doctor's communication were significantly more likely to be noncompliant with prescribed medications, as documented at a one-year follow-up.

A study conducted by Harpole et al (2003) from USA evaluated the impact of a headache management program 
that included structured individual and group sessions with a program manager. Fifty-four patients with chronic headache (61\% had a diagnosis of migraine) were referred to the program. As a group, these patients demonstrated a significant improvement in their headache-related disability, functional health status, and satisfaction with care over six months.

Blumenfeld and Tischio (2003) conducted a prospective cohort study of 422 patients with primary headache who participated in a headache management program within a multi-specialty medical group in USA. The headache management program included an educational session instructed by a neurologist and a nurse practitioner that was intended to improve communication, enhance patients' knowledge about their headache condition, and motivate patients to make appropriate life-style changes. Completion of the program was associated with significantly improved scores on the 36-item short form health survey and migrainespecific questionnaires, as well as a decrease in the number of primary care and emergency department visits. Ninety two percent of patients reported subjective improvement upon completion of the program. The degree to which the educational session contributed to these improved outcomes was not assessed.

\section{Discussion}

The impact of physician communication and patient understanding on patient outcomes has not been studied in migraine. The studies that we chose to describe provide indirect evidence to suggest that the quality of care for migraine can be improved with better patient understanding. One study (Lemstra et al 2002) highlighted the importance of a neurologist's advice and education. Another study (Holroyd et al 1989) demonstrated that an educational intervention that promoted self-management was associated with improved clinical outcomes. Several of the studies reviewed provide evidence that suggests patient satisfaction correlates with physician-patient communication or patients' understanding of their headache condition (Fitzpatrick and Hopkins 1981; Harpole et al 2003; Baos et al 2005; Campinha-Bacote et al 2005).

The role of physician communication and patient understanding has been assessed in other conditions (Ong et al 1995; Stewart 1995; Lewin et al 2001). The general conclusion of these studies is that improved physician communication and the provision of patient-centered care results in better patient satisfaction. Further, although it is difficult to quantify the benefits/effects of communication on patient health status, there is some evidence that suggests that better physician communication has a positive impact on health care outcomes as well (Greenfield et al 1985; Wiggers et al 1990; Simpson et al 1991; Stewart 1995).

Some, but not all, of the positive impact especially studied in diabetes, hypertension, asthma, and multiple sclerosis is due to enhanced medication adherence resulting from better communication (Kaplan et al 1989; Von Korff and Myers 1997; Gavin et al 1999; Ciechannowski 2001; Piette et al 2004; Thorne et al 2004).

Considering the fact that migraine patients are amongst the most dissatisfied with the medical care they receive, and that half of all patients with migraine who make the initial effort cease their quest for medical care, migraine may be the one for which improved physician communication might have the most significant public health impact $(\mathrm{Hu}$ et al 2000). Further, migraine is characterized by episodic events that must often be prevented or treated by the patient herself/ himself, usually without the benefit of physician at the time of the event. Patient understanding regarding the causes of, treatments for; and methods of prevention for migraine, is therefore critically important. Among other things, patients need to possess the knowledge about their migraine triggers, prodromal signs, healthful behaviors that can prevent frequent migraines, how to differentiate migraine from other possible headache etiologies, whether any medications are required, if medications are required which medications to take and when to take and when to repeat it, the exact dosage, what non-pharmacological approaches may be appropriate, when to seek medical attention for severe or "different" headaches, and how to avoid medication overuse/abuse and prevent transformation of episodic migraine to chronic daily headache.

Physicians and other health care providers are uniquely qualified to help educate their migraine patients in most of these areas. Such education is much more likely to be achieved with effective communication. Since evidence suggests that improvements in physician-patient communication could decrease the burden of suffering and health care resource utilization associated with other chronic conditions, it may be reasonable to assume that similar results can be expected in the outcomes of migraine. Further, since there is little evidence that low adherence with medications is disease- or regimen- specific (Haynes et al 2006), improved communication that results in better medication adherence can also be extrapolated to migraine.

In conclusion, despite the paucity of research that directly studies the impact of physician-patient communication in the management of migraine, the accumulation of indirect 
evidence suggests (albeit does not prove) the following links between improved patient-provider communication and outcomes of migraine:

- Improved patient-provider communication results in greater satisfaction with individual medical encounters;

- Over time, improved patient-provider communication results in increased patient knowledge and empowerment;

- Improved patient knowledge and outcome results in improved patient self-management; and

- Improved patient self-management results in improved satisfaction with medical care and improved clinical outcomes.

To these putative linkages we would also note that while the ultimate responsibility for improving patient-provider communication lies with the physician, time constraints in the clinical encounter suggest that much of this communication might be successfully delegated to other providers.

Future research priorities include making each of these linkages more direct, and in examining how non-physician providers can best be integrated into the care of patients with migraine.

\section{References}

Baos V, Ester F, Castellanos A, et al. 2005. Use of a structured migraine diary improves patient and physician communication about migraine disability and treatment outcomes. International Journal of Clinical Practice, 59:281-6.

Blumenfeld A, Tischio M. 2003. Center of excellence for headache care: group model at Kaiser Permanente. Headache, 43:431-40.

Campinha-Bacote DL, Kendle J, Jones C, et al. 2005. Impact of a migraine management program on improving health outcomes. Disease Management, 8:382-91.

Centonze V, Polito BM, Cassiano MA, et al. 1998. Patient education and migraine: a pilot study. Funct Neurol, 117-23.

Ciechanowski PS, Katon WJ, Russo JE, et al. 2001. The patient-provider relationship. Attachment theory and adherence to treatment in diabetes. American Journal of Psychiatry, 158:29-35.

Cottrell CK, Holroyd SE, Brose KA, et al. 2002. Perceptions and needs of patients with migraine: a focus group study. Journal of Family Practice, 51:142-7.

Edmeads J, Lainez JM, Brandes JL, et al. 2001. Potential of the Migraine Disability Assessment (MIDAS) questionnaire as a public health initiative and in clinical practice. Neurology, 56(6 Suppl 1):S29-34.

Fitzpatrick RM, Hopkins A. 1981. Patients' satisfaction with communication in neurological outpatient clinics. Journal of Psychosomatic Research, 25:329-34.

Foley MM, Carter-Edwards KA, Sueta L, et al. 2004. Disparities in lipid management for African Americans and Caucasians with coronary artery disease: a national cross-sectional study. BMC Cardiovascular Disorders, 4:15.

Foster KA, Liskin J, Cen S, et al. 2004. The Trager approach in the treatment of chronic headache: a pilot study. Alternative Therapies, 10(5):40-6.

Gavin LA, Sorokin N, Wamboldt MZ, et al. 1999. Treatment alliance and its association with family functioning, adherence, and medical outcome in adolescents with severe, chronic asthma. Journal of Pediatric Psychology, 24:355-65.
Greenfield S, Kaplan S, Ware JE Jr. 1985. Expanding patient involvement in care. Effects on patient outcomes. Annals of Internal Medicine, 102:520-8.

Harpole L, Samsa G, Jurgelski A, et al. 2003. Headache management program improves outcome for chronic headache. Headache, 43:715-24.

Haynes RB, Yao X, Degani A, et al. 2005. Interventions for enhancing medication adherence. The Cochrane Database of Systematic Reviews, Issue 4:CD000011.

Holroyd KA, Cordingley GE, Pingel JD, et al. 1989. Enhancing the effectiveness of abortive therapy: a controlled evaluation of self-management training. Headache, 29:148-53.

Hu XH, O’Donnell F, Kunkel RS, et al. 2000. Survey of migraineurs referred to headache specialists: care, satisfaction and outcomes. Neurology, 55:141-3.

Kaplan SH, Greenfield S, Gandek B, et al. 1996. Characteristics of physicians with participatory decision-making styles. Annals of Internal Medicine, 124:497-504.

Lemstra M, Olszynski WP, Stewart B. 2002. Effectiveness of multidisciplinary intervention in the treatment of migraine: a randomized clinical trial. Headache, 42:845-54.

Lewin SA, Skea ZC, Entwistle V, et al. 2001. Interventions for providers to promote a patient-centered approach in clinical consultations. Cochrane Database of Systematic Reviews, Issue 4:CD003267.

Lipton RB, Diamond S, Reed M, et al. 2001. Migraine diagnosis and treatment: results from the American Migraine Study II. Headache, 41:638-45.

Lipton RB, Stewart WF, Diamond S, et al. 2001. Prevalence and burden of migraine in the United States: data from American Migraine Study II. Headache, 41:646-57.

Lipton RB, Stewart WF, Simon D. 1998. Medical consultation for migraine: results from the American Migraine Study. Headache, 38:87-96.

McGarth P, Humphreys P, Keene D, et al. 1992. The efficacy and efficiency of a self-administered treatment for adolescent migraine. Pain, 49:321-4.

Nicholson R, Nash J, Andrasik F. 2005. A self-administered behavioral intervention using tailored messages for migraine. Headache, 45:1124-39.

Ong LM, de Haes JC, Hoos AM, et al. 1995. Doctor-patient communication: a review of the literature. Social Science and Medicine, 40:903-18.

Piette JD, Heisler M, Wagner TH. 2004. Cost-related medication underuse among chronically ill adults: the treatments people forgo, how often, and who is at risk. American Journal of Public Health, 94:1782-7.

Rothrock JF, Parada VA, Sims C, et al. 2006. The impact of intensive patient education on clinical outcome in a clinic-based migraine population. Headache, 46:726-31.

Silberstein SD, Rosenberg J. 2000. Multispecialty consensus on diagnosis and treatment of headache. Neurology, 54:1553-4.

Simpson M, Buckman R, Stewart M, et al. 1991. Doctor-patient communication: the Toronto consensus statement. British Medical Journal, 303:1385-7.

South V, Sheftell F. 2001. Communicating with the patient. In: Silberstein SD, Lipton RB, Dalessio DJ eds. Wolff's headache and other head pain. (7th ed.) New York, NY: Oxford University Press. p 599-606.

Stewart MA. 1995. Effective physician-patient communication and health outcomes: a review. Canadian Medical Association Journal, 152:1423-33.

Stewart WF, Lipton RB, Celentano DD, et al. 1992. Prevalence of migraine headache in the United States. Relation to age, income, race and other socio-demographic factors. JAMA, 267:64-9.

Thorne SE, Harris SR, Mahoney K, et al. 2004. The context of health care communication in chronic illness. Patient Education and Counseling, 54:299-306.

Von Korff M, Myers L. 1987. The primary care physician and psychiatric services. General Hospital Psychiatry, 9:235-40.

Wiggers JH, Donovan KO, Redman S, et al. 1990. Cancer patient satisfaction with care. Cancer, 66:610-6. 
